\title{
A Remote Sensing Scheme for Fault Diagnosis to Wind Turbines: An Academic Experimental Set-Up
}

\author{
L. Acho ${ }^{1}$ \\ ${ }^{1}$ Department of Mathematics \\ U.P.C., Polytechnic University of Catalunya \\ Campus of Terrassa - Barcelona, 08222 BCN (Spain) \\ e-mail: leonardo.acho@upc.edu
}

\begin{abstract}
Nowadays, remote sensing for structural fault examination to wind turbines is an important technological challenger. On the other hand, laser diodes represent a low-cost option to implement a remote vibrometer sensor by just using cheap analog electronics. Therefore, a recent electronic circuit along with a laser diode is conceived to detect faults on a winding structure due to vibrations mainly induced by the wind and its rotary parts with possible mechanical defects. The electronic parts consist of a sequence of bandpass filters and peak detector stages. Besides, an academic experimental platform is constructed to validate the performance of the proposed remote sensing scheme for fault diagnosis in wind turbine structures.
\end{abstract}

Key words. Wind turbines, fault analysis, electronics, lasers.

\section{Introduction}

Lately, there have been reported many approaches on fault detection to wind turbine structures [1-3]. Some of them are based on intelligent computation [4,5], others on observers and control techniques [6,7], and others on image processing [8]; and so on [9]. In contrast, the design of fault diagnosis methods to these systems by using lowcost electronic components is not completely developed but some few reported (see a patent here). Hence, the main objective of this presentation is to conceive a remote sensing fault diagnosis approach to wind turbinesstructures by employing low-cost analog electronics and a laser diode, which is also a cheap electronic element. To validate this design, a simple experimental platform is mounted to emulate vibrations on a wind turbine structure, see Figure 1. Here, by varying the load mass on the rotational flexible bar, different fault cases may be emulated. And the unloaded case can be considered as a healthy operation. Additionally, the employed drill machine has its own control angular speed with the reference command given by the user. This facilitates preparation of a set of real scenarios on experimentation. Then, the laser diode and its instrumentation; and the electronic circuit to generate the tracer signals $\mathrm{M}_{1}, \mathrm{M}_{2}$, and $\mathrm{M}_{3}$ are implemented. The electronics to generate the tracer signals are basically composed of a set of tuned bandpass filters and peak detector stages. All the proposed electronics fault detection schemes are realized by using analog electronics. Therefore, the tracer signals are produced in real-time, which, in contrast to other computational techniques $[8,10]$, does not require extra time on fault diagnosis. Besides, this experimental platform may be proactive to some engineering lectures on electronics and signal processing topics to wind turbines. Therefore, the following paper contributions are evidenced:

- A Recent electronics to generate trace signals able to discriminate different scenarios of a structure under vibration.

- A low-cost electronics approach on wind turbine structures for remote fault diagnosis in real-time monitoring.

- A low-cost experimental platform for engineering on electronics and signal processing lectures.

The rest of the paper is structured as follows. Section 2 introduces the electronics instrumentation to the laser diode for the remote vibrometer design. Section 3 describes the electronics involved to state the generator tracer signals approach. Section 4 shows the experimental result for the healthy and faulty cases for different angular values of an emulated blade system. Finally, final remarks are written in Section 5.

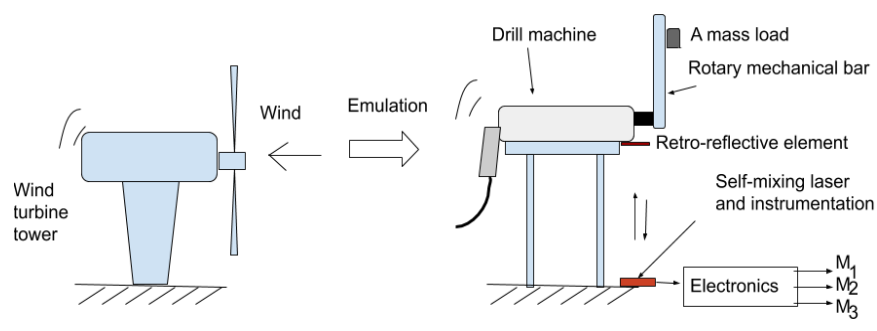

Fig.1. Experimental platform. The output signals $\mathrm{M}_{1}, \mathrm{M}_{2}$, and $\mathrm{M}_{3}$ are used as tracer signals. The input signal to this block comes from the laser housing. The rotary flexible bar is the main source of vibrations, and faults are captured by changing its mass load body. 


\section{Remote vibration measurement based on laser diodes}

This section introduces the implemented remote vibration sensor based on a laser diode as the transmitter, and a photo-resistor as the receiver. The photo-resistor varies its resistance between $1.5 \mathrm{M} \Omega$ to $3 \mathrm{k} \Omega$ from a darkness condition to a normal office room illumination, respectively. On the other hand, the used laser diode is the one commonly used by the Arduino Uno community. This corresponds to the KY-008 class. Therefore, the beam is retro-reflected to the photo-resistor by the reflective element attached to the structure under vibration. See Figures 2 and 1. And under a vibration on the structure, the reflected laser beam will vary its incidence on the photo-resistor producing a lecture signal in accordance with the structure vibration.

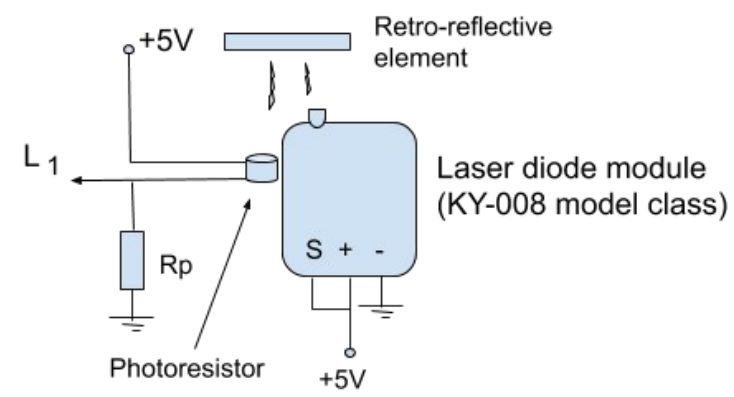

Fig. 2 Laser diode housing for remote vibration monitoring. $L_{1}$ is the sensor lecture to the electronics section (See Figure 1). Here $\mathrm{R}_{\mathrm{P}}=10 \mathrm{k} \Omega$. The laser beam is transmitted by the laser diode module and then reflected to the photoresistor by the retroreflective element.

\section{Electronics for generating the tracer signals}

This written part is dedicated to showing the electronics to produce the tracer signals needed for fault diagnosis to the experimental platform, where this platform was conceived to emulate a wind turbine on operation. Due to the photoresistor in the remote vibration sensor is sensitive to the ambient light, and in order to just capture the best as possible the fast-changing time variation induced by vibration, a high pass filter (HPF) is invoked. Then, a low pass filter (LPF) followed by a peak detector both are constructed. See Fig. 3. In this way, a kind of a nonlinear bandpass filter is obtained.

In the average sense, a large-scale wind turbine has its low rotational shaft connected to a gearbox to increase the rotational speed of the rotational shaft of the electrical generator to about $1200-1800 \mathrm{rpm}$. On the other hand, a common ratio of the gearbox is about 90:1. Hence, the low rotational shaft speed is located between 13.3 to 20 rpm. Additionally, if a mechanical fault is present, a vibration frequency content will be beyond marked by the $20 \mathrm{rpm}$. Therefore, for experimentation, we set the vibration frequency content in the interval from 12 to 950 $\mathrm{rad} / \mathrm{s}$. Then, this interval is separated into three intervals to produce the electronics on the trace signals generator as shown in Fig. 4.

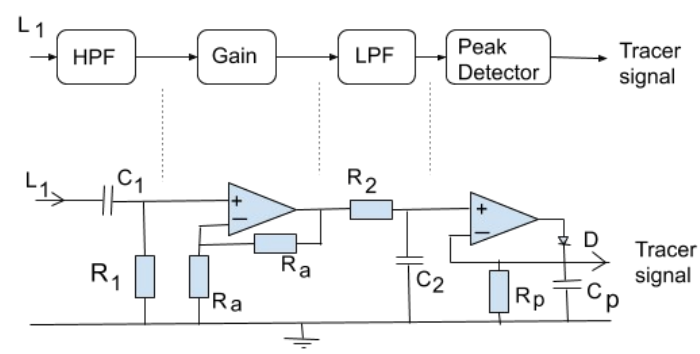

Fig. 3 Electronics realization of a tracer signal by using the vibration sensor lecture. $\mathrm{w}_{1}=1 /\left(\mathrm{R}_{1} \mathrm{C}_{1}\right)$ and $\mathrm{w}_{2}=1 /\left(\mathrm{R}_{2} \mathrm{C}_{2}\right)$ are the corresponding cut-off frequencies of the stated filters (in $\mathrm{rad} / \mathrm{s}$ ), and the gain is set to 2 . Additionally, the peak detector module is composed of a rectifier diode $\mathrm{D}$.
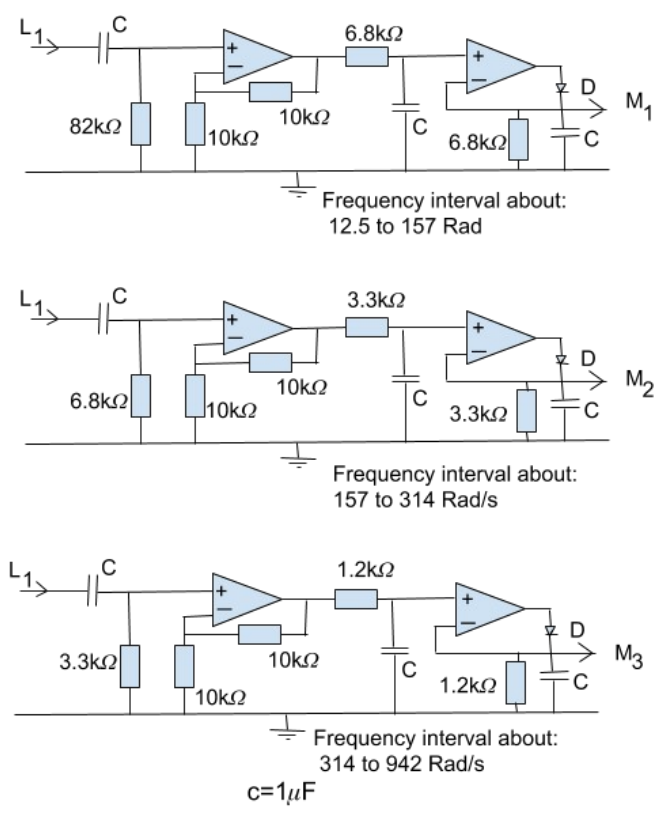

Fig. 4 Electronics for the tracer signals generator. The operational amplifiers are instrumented by using the LM741 and supplied by a dual DC power source of 12 volts. The diode rectifier is the $1 \mathrm{~N} 4001$ each.

\section{Experimental platform and results}

To complete the whole experimental realization, Figures 5 to 8 show a set of pictures of the experimental platform. There, a drill machine is used to produce a rotational movement. And the bar with a mass load is to emulate the wind turbine blades in motion. Additionally, this drill has a rotational speed control set by the user. It also states a lecture on the programmed rotational speed. This kind of control may emulate a rotational speed control in a wind turbine in the called Region 3 (a wind turbine mode operation for rotational speed regulation control). 

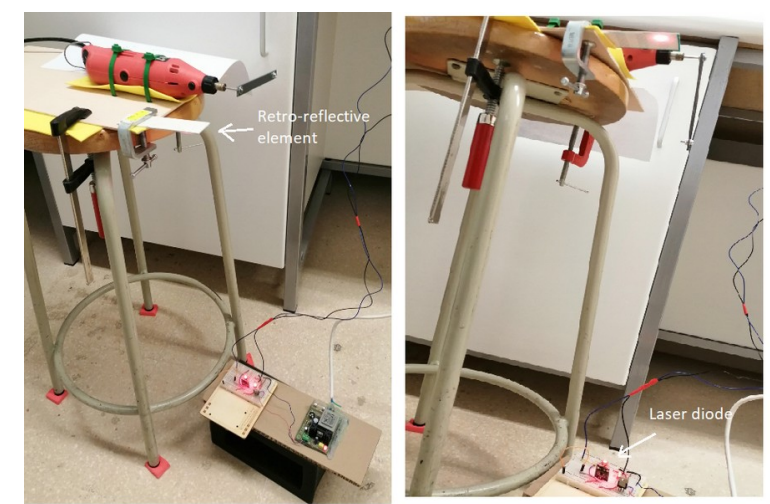

Fig. 5 Experimental platform showing the drill machine, the reflective element, the laser diode, and the flexible bar without a load mass.

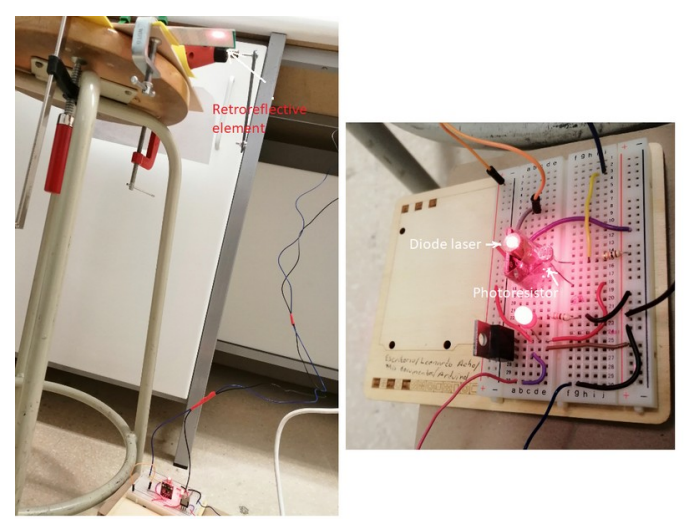

Fig. 6 Images showing the laser beam and the retro-reflective element in action.
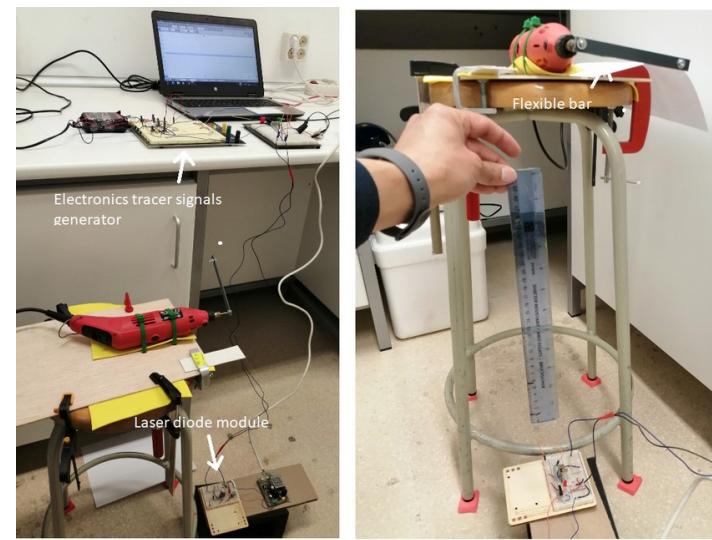

Fig. 7 Pictures evidencing the experimental platform and a computer for data acquisition results.

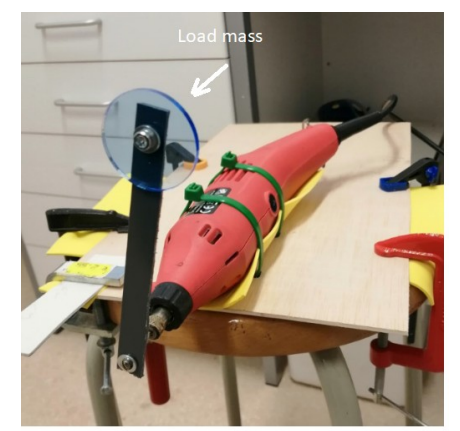

Fig. 8 The experimental set-up with a mass load of 0.7 grams.
Then, the experimental platform was operated at $800 \mathrm{rpm}$ and $1200 \mathrm{rpm}$, for the no-load mass, and again repeated for a load case (see Figure 8). Therefore, the unloaded case may emulate a healthy operation of the wind turbine, and the load case may emulate a structure fault, for instance, on the wind turbine blades.

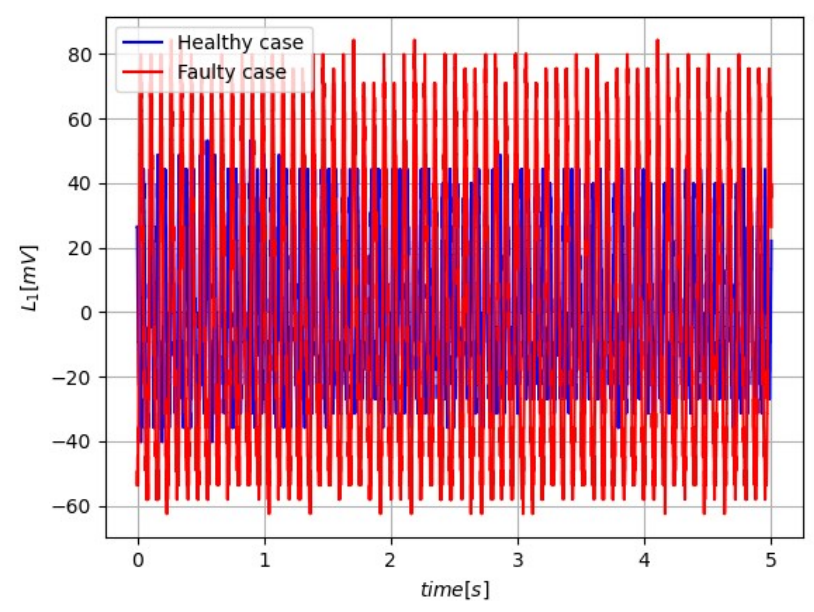

Fig. 9 Lecture of the signal $\mathrm{L}_{1}$ given by the remote vibrometer when the platform is operated at $800 \mathrm{rpm}$ for both cases, the load (Faulty) and no load (Healthy) cases.

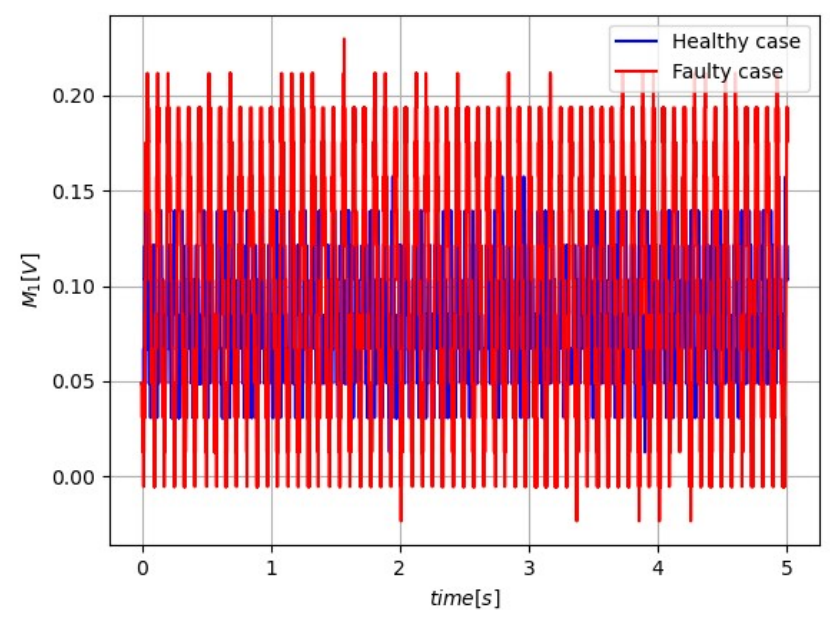

Fig. 10 Lecture of the signal $\mathrm{M}_{1}$ produced by the electronics stage when the platform is operated at $800 \mathrm{rpm}$ for both cases, the load (Faulty) and no load (Healthy) cases. 


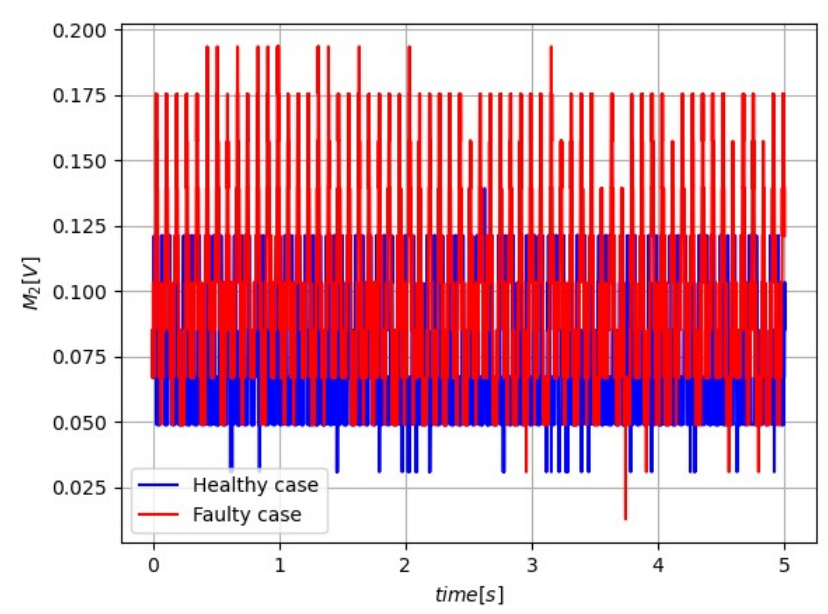

Fig. 11 Lecture of the signal $\mathrm{M}_{2}$ generated by the electronics stage when the platform is operated at 800rpm for both cases, the load (Faulty) and no load (Healthy) cases.

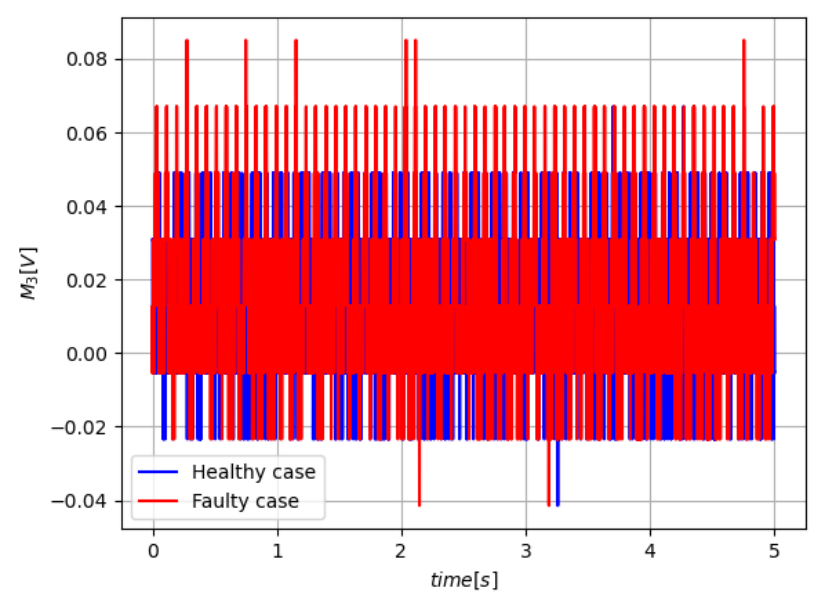

Fig. 12 Lecture of the signal $\mathrm{M}_{3}$ supplied by the electronics stage when the platform is operated at $800 \mathrm{rpm}$ for both cases, the load (Faulty) and no load (Healthy) cases.

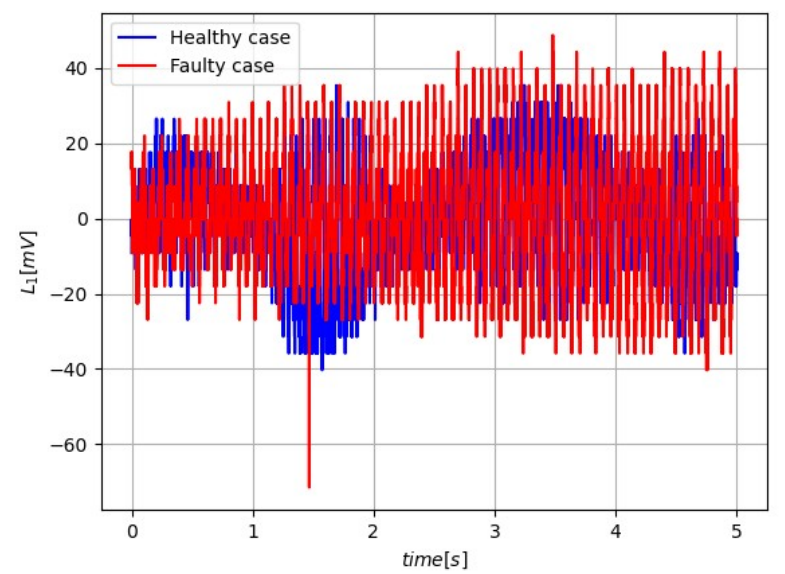

Fig. 13 Lecture of the signal $\mathrm{L}_{1}$ given by the remote vibrometer when the platform is operated at $1200 \mathrm{rpm}$ for both cases, the load (Faulty) and no load (Healthy) cases.

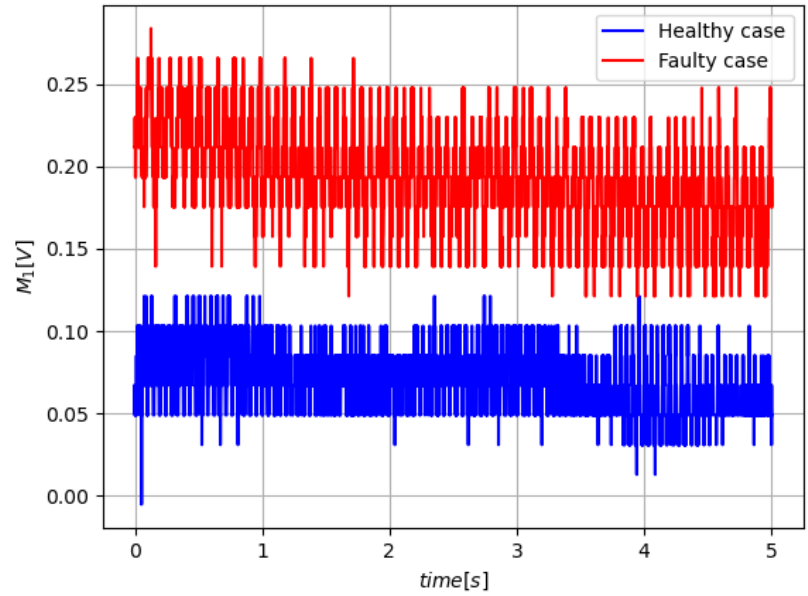

Fig. 14 Lecture of the signal $\mathrm{M}_{1}$ produced by the electronics stage when the platform is operated at 1200rpm for both cases, the load (Faulty) and no load (Healthy) cases.

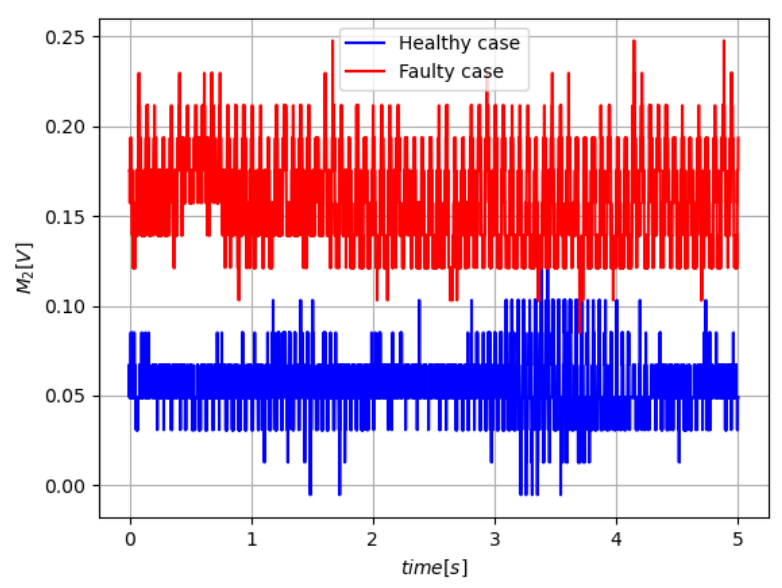

Fig. 15 Lecture of the signal $\mathrm{M}_{2}$ generated by the electronics stage when the platform is operated at 1200rpm for both cases, the load (Faulty) and no load (Healthy) cases.

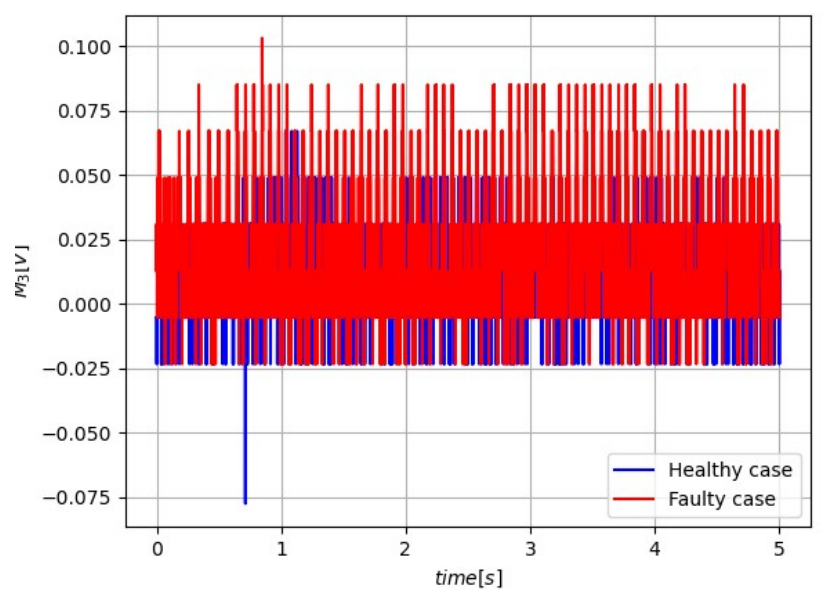

Fig. 16 Lecture of the signal $\mathrm{M}_{3}$ supplied by the electronics stage when the platform is operated at $1200 \mathrm{rpm}$ for both cases, the load (Faulty) and no load (Healthy) cases. 
From the previous experimental results, we can note that the electronics employed to generate the tracer signals, these are able to discriminate between the faulty and healthy cases. Obviously, in our experimental set, just two cases were analyzed at two different angular speed values, and more experiments are required to run. But, the main issue on diagnosis is realized in real-time, and it can be improved, for instance, by employing analog electronics threshold detectors, or others, which results inexpensive too. All these subjects are left for future research. Finally, to highlight that the experimental results were captured by using a digital oscilloscope (the PicoScope one, model 2204-A), where for the case of $\mathrm{L}_{1}$ was captured in the CA mode and the others in the $\mathrm{CC}$ mode.

\section{Conclusion}

A low-cost experimental platform was realized by using laser technology. The main advantage of this technology is its ability for remote monitoring and contactless too. Additionally, and due to analog electronics being involved in the design, the signal processing is realized online. One main disadvantage is the careful calculation of the electronic circuits-parameters, and ambient light may require to be insulated from the photo-resistor device. As a final discussion, and by following the reviewers' comments, some facts are introduced:

- For calibration of the proposed electronic system, just the filters' frequencies are the important ones. Obviously, by introducing some potentiometers, a final adjustment can be tested online.

- There was a reviewer comment about that using digital electronics and the SCADA system of a wind turbine will be much better than the analog electronic version of the given approach. This is true depending on the expertise field. Nowadays, all the electronics are moving into the digital electronic world, but an analog electronic design is always a cheap option to test new dynamical system realization. For instance, to develop the present work, a SCADA system was not available for us. But you can always move from the analog version to the digital one.

- There was a reviewer observation on the fact that we are using 'local' information instead of remote sensing. In our approach, the signals are measured at the ground of the wind tower and reading, remotely, vibrations on the top of the tower. This is not a limitation. Just to recall, NASA is measurements the moon's vibration by using a laser located on earth and by just using a mirror on the moon's surface. Of course, they used a square reflective one. This mirror always reflects the laser beam to the beam source wherever is its location.

\section{Acknowledgement}

This research was completely funded by the Spanish Ministry of Economy and Competitiveness (State Research Agency of the Spanish Government)/Fondos Europeos de Desarrollo Regional (MINECO/FEDER), grant number DPI2015-64170-R.

\section{References}

[1] Luo, N.; Vidal, Y.; Acho, L. Wind turbine control and monitoring; Springer, Berlin (2014).

[2] Ahadi, A. "Wind turbine fault diagnosis techniques and related algorithms". International Journal of Renewable Energy Research -IJRER (2016), Vol. 6, pp. 80-89.

[3] Maldonado-Correa, J.; Martín-Martínez, S.; Artigao, E.; Gómez-Lázaro, E. "Using SCADA Data for Wind Turbine Condition Monitoring: A Systematic Literature Review". Energies (2020), Vol. 13, pp. 3132.

[4] Tang, B.; Liu, W.; Song, T. "Wind turbine fault diagnosis based on Morlet wavelet transformation and Wigner-Ville distribution". Renewable Energy (2010), Vol. 35, pp. 2862 2866.

[5] Wenyi, L.; Zhenfeng, W.; Jiguang, H.; Guangfeng, W. "Wind turbine fault diagnosis method based on diagonal spectrum and clustering binary tree SVM". Renewable Energy (2013), Vol. 50, pp. 1-6.

[6] Vidal, Y.; Tutivén, C.; Rodellar, J.; Acho, L. "Fault diagnosis and fault-tolerant control of wind turbines via a discrete time controller with a disturbance compensator". Energies (2015), Vol. 8, pp. 4300-4316.

[7] Pujol-Vazquez, G.; Acho, L.; Gibergans-Báguena, J. "Fault Detection Algorithm for Wind Turbines' Pitch Actuator Systems". Energies (2020), Vol. 13, pp. 2861.

[8] Ruiz, M.; Mujica, L.E.; Alferez, S.; Acho, L.; Tutiven, C.; Vidal, Y.; Rodellar, J.; Pozo, F. "Wind turbine fault detection and classification by means of image texture analysis". Mechanical Systems and Signal Processing (2018), Vol. 107, pp. 149-167.

[9] Jiang, G.; Xie, P.; He, H.; Yan, J. Wind turbine fault detection using a denoising autoencoder with temporal information. IEEE/Asme transactions on mechatronics (2017), Vol. 23, pp. 89-100.

[10] Wang, Y.; Ma, X.; Qian, P. "Wind turbine fault detection and identification through PCA-based optimal variable selection". IEEE Transactions on Sustainable Energy (2018), Vol. 9, pp. 1627-1635. 